\title{
Earthen Levee Monitoring with Synthetic Aperture Radar
}

\author{
James V. Aanstoos, Khaled Hasan, Charles G. O’Hara, Saurabh Prasad, Lalitha Dabbiru, Majid Mahrooghy, \\ Balakrishna Gokaraju, Matthew Lee, Rodrigo Nobrega \\ Geosystems Research Institute \\ Mississippi State University \\ Mississippi State, MS 39762 USA \\ j.aanstoos@ieee.org
}

\begin{abstract}
The latest results are presented from an ongoing study of the use of multi-polarized Synthetic Aperture Radar as an aid in screening earthen levees for weak points. Both L-band airborne and $X$-band spaceborne radars are studied, using the NASA UAVSAR and the German TerraSAR-X platforms. Feature detection and classification algorithms tested for this application include both radiometric and textural methods. Radiometric features include both the simple backscatter magnitudes of the $\mathrm{HH}, \mathrm{VV}$, and $\mathrm{HV}$ channels as well as decompositions such as Entropy, Anisotropy, and Alpha angle. Textural methods include grey-level co-occurrence matrix and wavelet features. Classifiers tested include Maximum Likelihood and Artificial Neural Networks. The study area includes $240 \mathrm{~km}$ of levees along the lower Mississippi River. Results to date are encouraging but still very preliminary and in need of further validation and testing.
\end{abstract} levees

Keywords-synthetic aperture radar, levee screening, earthen

\section{INTRODUCTION}

Earthen levees protect large areas of populated and cultivated land in the US from flooding. In the United States there are more than 150,000 kilometers of levee structures of varying designs and conditions. The potential loss of life and property associated with the catastrophic failure of levees can be extremely large.

Currently, there are limited processes in place to prioritize the monitoring of large numbers of dam and levee structures. . There is a need to prioritize the monitoring of the network of dam and levee structures. Levee managers and federal agencies will benefit from any tools allowing them to assess levee health rapidly with robust techniques that identify, classify and prioritize levee vulnerabilities with lower costs than traditional programs not based on the use of remote sensing. This paper presents early results of an ongoing project studying the use of synthetic aperture radar (SAR) as an aid to the levee screening process. Sources of data include:

(1)

\begin{abstract}
The NASA UAVSAR (Uninhabited Aerial Vehicle SAR), a fully polarimetric L-band SAR which is specifically designed to acquire airborne repeat track SAR data for differential interferometric measurements. This instrument is capable of submeter ground sample distance.
\end{abstract}

Funded in part by Department of Homeland Security Southeast Region Research Initiative (SERRI) at Department of Energy's Oak Ridge National Laboratory, and by NASA's Applied Sciences Program
The Gerrman TerraSAR-X satellite with its X-band multi-polarimetric SAR and high spatial resolution

The longer-wavelength L-band SAR measurements can penetrate vegetation and even the top layer of soil, depending on moisture content. On that basis this wavelength was selected for our study under the assumption that the backscatter will carry information from the top layer of the soil that will be valuable in detecting changes in levees that will be key inputs to a levee vulnerability classification system.

The TerraSAR-X satellite provides advantages of better temporal resolution and lower cost of data acquisitions than an airborne platform in general, and also high spatial resolution. The shorter wavelength however results in less penetration depth, especially in the presence of vegetation such as trees, shrubs and grass, thus reducing the amount of information about the soil present in the backscatter. However, some variations in the vegetation itself may be related to levee vulnerabilities, mitigating this disadvantage of the shorter wavelength option.

Our test study area is a stretch of $240 \mathrm{~km}$ of levees along the lower Mississippi River, along the western boundary of the state of Mississippi. Two types of problems that occur along these levees which can be precursors to complete failure during a high water event are sand boils and slough (or slump) slides.

Sand boils are springs that form on the land side of a levee which is containing a river at extremely high flood stage. Hydrostatic pressure generated by the column of river water exerts a downward force that is too great for the wall material of the river channel to contain, and thus water is forced through the wall material of the channel. [1]

Slough slides are slope failures along a levee, which leave areas of the levee vulnerable to seepage and failure during high water events. A schematic illustration of slides and sand boils is shown in Fig. 1 [2]. A photograph of a typical slough slide, in this case one which appeared during the recent spring 2011 flooding of the Mississippi River, is shown in Fig. 2. The roughness and related textural characteristics of the soil in a slide affect the amount and pattern of radar backscatter. The type of vegetation that grows in a slide area differs from the surrounding levee vegetation, which can also be utilized in detecting slides [3]. 
Early detection of the occurrence of slides and boils could help levee mangers prioritize their inspection and repair efforts. A remote sensing based solution for their rapid detection would be more efficient and cost effective than frequent on-site visits. Furthermore, it may be possible to detect less obvious precursors to slides and boils by sensing characteristics of the surface soils and vegetation. A working hypothesis of this study is that such characteristics will be manifested in the backscatter of polarimetric radar due to its response to spatially variant soil moisture. For example, $\mathrm{L}$ band radar is known to penetrate dry soils up to one meter in depth, and has been used to map surface soil moisture. [4] Although our ultimate goal is classification of vulnerable levee segments, we are also testing the ability to map surface soil moisture patterns since such patterns can serve as a feature useful for the levee classification. Furthermore, comparing soil moisture to radar signatures in our study area gives us much more reference data to train and test on, versus limiting the study to slump slides and boils.

\section{DATA}

\section{A. UAVSAR Data}

The primary remotely sensed data used in this study is from NASA Jet Propulsion Laboratory's UAVSAR (Uninhabited Aerial Vehicle Synthetic Aperture Radar) instrument, a polarimetric L-band synthetic aperture radar flown on a Gulfstream-3 research aircraft. The salient characteristics of this instrument are shown in Table I.

The UAVSAR is normally flown at an altitude of $12.5 \mathrm{~km}$ and takes an image swath $20 \mathrm{~km}$ wide. Our study area was designed to be collected in two straight-line flight segments structured to capture most of the river levees on both sides of the river. The study area is shown in Fig. 3, with a color composite representation of the radar data overlaid on a base map of the vicinity. A total of five UAVSAR data collections have been made: (1) June 16, 2009; (2) January 25, 2010; (3) April 28 2010; (4) June 7, 2011; and (5) June 22, 2011. The higher frequency of flights during the spring of 2011 was driven by the 100-year level flood event occurring at that time, which created a valuable opportunity for collecting data at very high-water levels. The flights were flown in a "racetrack" pattern looking toward the river from opposite directions, in order to achieve a range of local incidence angles along the levees.

TABLE I. UAVSAR SPECIFICATIONS [5]

\begin{tabular}{|r|l|}
\hline \multicolumn{1}{|c|}{ Parameter } & \multicolumn{1}{|c|}{ Value } \\
\hline Frequency & L-band \\
\hline Bandwidth & $80 \mathrm{MHz}$ \\
\hline Range Resolution & $1.8 \mathrm{~m}$ \\
\hline Polarization & Full quad polarization \\
\hline Quantization & 12 bits \\
\hline Antenna size & $0.5 \mathrm{~m}$ range/1.5 azimuth \\
\hline Power & $>2.0 \mathrm{~kW}$ \\
\hline
\end{tabular}

Although the raw ground sample distance is 1.6 by 0.6 meters, most of our efforts use the multi-look 5 by 7 meter data to minimize speckle effects.

\section{B. Satellite Data}

In addition to UAVSAR, data from the German TerraSAR$\mathrm{X}$ satellite was acquired over portions of the same study area. Characteristics of this sensor are shown in Table II. Due to the relatively low cost (for TerraSAR-X Science Team members), we acquired a large number of such scenes over a great variety of seasons and time periods. As of now, a total of 34 TerraSAR scenes have been acquired. The locations and scene sizes relative to the UAVSAR image swath are shown in Fig. 4.

TABLE II. TERRASAR-X SPECIFICATIONS [6]

\begin{tabular}{|r|l|}
\hline Parameter & \multicolumn{1}{|c|}{ Value } \\
\hline Radar Carrier Frequency & $9.65 \mathrm{GHz}$ (X-band) \\
\hline Bandwidth & $150 \mathrm{MHz}$ \\
\hline $\begin{array}{r}\text { Pulse Repetition } \\
\text { Frequency }\end{array}$ & $2-6.5 \mathrm{KHz}$ \\
\hline Incidence Angle Range & $20^{\circ}-45^{\circ}$ \\
\hline Polarization & $\mathrm{HH}, \mathrm{HV}, \mathrm{VH}, \mathrm{VV}$ \\
\hline Nominal altitude & $514 \mathrm{~km}$ \\
\hline Revisit time & 11 days \\
\hline Power & $>2.0 \mathrm{~kW}$ \\
\hline
\end{tabular}

\section{Ancillary and Reference Data}

Ancillary data can be used to assist the levee classification process in addition to the remote sensing data. This includes knowledge of the soil characteristics in the vicinity of the levees, the underlying geology of the area, and history of past problems and inspections of the levees.

Reference (or "ground truth") data is obtained for the purpose of training the supervised classification algorithms and testing and validation of results. Such data falls into two major categories: (1) known levee vulnerability points such as slump slides, seepage points, or sand boils; and (2) measured soil properties such as moisture content, sand/clay ratios, etc. The former category is collected with the assistance of the US Army Corps of Engineers (USACE), which maintains a good cumulative history of past problems and has identified particularly problematic sections of levees in the study area. A challenge in using this data is that once USACE identifies problems it soon repairs them, depending on their severity. This leaves the targets of interest in their natural (un-repaired) state for a limited period of time, making the number of such targets available during a given remote sensing data acquisitions relatively small. On the assumption that the soil properties in the vicinity of such problems may have similar characteristics which may be detectable in the radar signatures, we use some of these repaired locations in our training data, and plot their locations along with the unrepaired targets when analyzing results.

The second category of reference data, measured soil properties is collected in a number of ways. We make direct measurements of soil moisture content (volumetric water content $(\% \mathrm{VWC})$ using handheld probes during each radar 
flight or satellite overpass. We focus these collections on specific areas of interest (AOIs) within the large study are, which include slide and boil regions. In addition we contracted with a company (Soil and Topography Information, LLC) that performs intelligent sampling collection of soil property measurements using vehicle-based probes that allow a large collection area efficiently. We measured in this manner soil properties over a 3-mile long section of the study area levees, which included our other AOIs. An example of this data is shown in Fig. 5. Finally, our partners at the USACE also collected electromagnetic conductivity (EC) soil measurements over an intermediate sized AOI during some of the radar collection times. These serve as a good proxy for direct soil moisture data.

\section{ClassificAtion Methodology}

Features believed to provide good potential for discrimination of the targets of interest were designed, computed, and tested. Both per-pixel and window-based (textural) features were examined. The candidate features were tested with our training data to determine separability between classes of interest. Fig. 6 shows the overall approach to the classifier design. Over 144 features are investigated, ig radiometric and textural features (described below). Classification algorithms tested include a back-propagation Artificial Neural Network (ANN) and the Maximum Likelihood classifier.

Stepwise Linear Discriminant Analysis (S-LDA) is employed for feature reduction and optimization [7]. In this approach, various features derived from the SAR backscatter imagery are concatenated into a vector, and a forwardselection, backward rejection technique is employed to prune away features that are "less" relevant to the classification problem at hand. S-LDA reduces the feature set by selecting a subset of all available features based on a metric that quantifies the class separation provided by each feature. In this study Bhattacharyya Distance (BD) is used as the metric for calculating the class separation. An LDA based feature "optimization" is then employed on this reduced dimensional subset of features identified by the forward selection, backward rejection search. S-LDA is hence extremely valuable in ensuring that the "most" relevant features are provided to the classifier while ensuring that the classifier is not over-burdened by an excessively high dimensional feature space. he "classifier" employed in this work to model class-specific information and label test data (pixels) appropriately is the popular maximum-likelihood classifier. This classifier assumes Gaussian probability distribution functions for each class, and uses training data to learn the mean vector and covariance matrix per class. This information is then employed to find the distance of test vectors from each class model, and a class label is assigned to the sample that maximizes the likelihood value of the test sample being in that class.

Features included in this study are described in detail below. These include per-pixel intensity and related features, as well as features extracted within a window around each pixel known as textural features.

\section{A. Per-pixel Features}

The polarimetric radar data contain three independent channels of backscatter coefficients, those for like-polarized $(\mathrm{HH}, \mathrm{VV})$ and cross-polarized (HV) combinations of transmitted and received polarizations. For each, we get complex values giving both magnitude and phase information. The magnitudes of these channels can be used as basic perpixel features with any classifier. For the airborne UAVSAR radar data we have two different views of the same levees from opposite directions and have used the 3 channels from each direction for each pixel in case there is additional information based on local incidence angle.

The relationship between the complex backscatter coefficients can reveal details on the nature of the scattering mechanism of the targets, such as relative amount of surface, double-bounce, or volume scattering. Both supervised and unsupervised classification techniques are used with the polarimetric decomposition parameters entropy $(\mathrm{H})$, anisotropy (A) and alpha $(\alpha)$ derived from the coherency matrix calculated for each pixel [8]. Though these features are also per-pixel and derived from the polarimetric channels, their closer relationship to physical scattering mechanisms may make them more useful in identifying surface anomalies such as slump slides.

In addition, since models have been developed for estimating soil moisture from SAR images, we implemented these to determine if the resulting patterns of moisture estimates on the levees can be used as a classification feature. Oh et al. [9] developed an inversion technique to obtain surface roughness and dielectric constant (related to water content) from the co-polarization ratio $\mathrm{p}$ and the cross-polarization ratio q. The model works over limited ranges of roughness and moisture. The co-polarization and cross-polarization ratios are defined as:

$$
p=\sigma_{h h}^{0} / \sigma_{v v}^{0} \quad q=\sigma_{h v}^{0} / \sigma_{v v}^{0}
$$

where $\sigma_{h h}^{0}$ is the HH polarized backscatter coefficient, $\sigma_{v v}^{0}$ the VV polarized backscatter coefficient, and $\sigma_{h v}^{0}$ the $\mathrm{HV}$ polarized backscatter coefficient. The dielectric constant is obtained by the following empirical equations: [10]

$$
\begin{aligned}
& \left(\frac{2 \theta}{\pi}\right)^{1 / 3 \Gamma_{0}} \cdot\left[1-\frac{q}{0.23 \sqrt{\Gamma_{0}}}\right]+\sqrt{p}-1=0 \\
& \Gamma_{0}=\left|\frac{1-\sqrt{\varepsilon_{r}}}{1+\sqrt{\varepsilon_{r}}}\right|^{2}
\end{aligned}
$$

where $\theta$ is the incidence angle in radians, $\Gamma_{0}$ is the Fresnel reflectivity of the surface at nadir and $\varepsilon_{\mathrm{r}}$ is the real part of dialectic constant (the imaginary part is ignored).

In addition to directly implementing this empirical moisture model, we have tested a back propagation ANN for moisture classification. Results of these studies are reported elsewhere [11]. 


\section{B. Textural Features}

In addition to the per-pixel backscatter features, a number of texture features are being explored which utilize the values from pixels in a neighborhood around each pixel being analyzed. Features based on the Gray Level Co-occurrence Matrix (GLCM) [12] and on the discrete wavelet transform (DWT) [13], continue to show promising results.

The GLCM methods use estimates of the joint probability distribution of pixel pairs at given distances in given directions. Summary statistics are then calculated on the resulting NxN cooccurrence matrices, where $\mathrm{N}$ is the number of quantized gray levels - that is, intensity ranges for a given channel of image data (we use $\mathrm{N}=256$ ). The summary statistics can include such measures as mean, variance, correlation, energy, homogeneity, anisotropy and entropy.

The ability of wavelet analysis to decompose an image into different frequency sub-bands makes it suitable for image classification [13] [14]. In some applications, the energy of each sub-band is used as a texture feature. In others, a feature selection analysis chooses a subset of these which prove effective for a given texture-based classification. Other parameters to be determined include the choice of mother wavelet function, and the neighborhood window size.

For our application, we used wavelet features with one decomposition level from each of the radar polarization channels. We tested these features using different sliding window sizes $(5,7,8,9)$ and mother wavelets (Daubechies, Haar, Symlet, and Biorthogonal) and selected those that maximize the separation of the targets of interest from the background [15].

\section{RESULTS}

Results of this ongoing study, which must still be considered preliminary, continue to show promise.

Some results of using the S-LDA feature selection method on our texture features are shown in Fig. 7. This shows the result of classifying a subset of our study area which contains two slough slides using a 2-class problem (slide or normal levee). The S-LDA algorithm selected the two best features for this example, and those were both based on the $\mathrm{HH}$ radar data channel: (1) the GLCM entropy measure from a 135 degree direction, and (2) the variance of the $5 \times 5$ pixel window. Many of the patches of pixels identified as slides do in fact correspond to actual slides. Most of the false positives have region shape characteristics that can be used to filter them out using morphological operators: for example, the isolated smaller (often single-pixel) false positives in the figure could easily be eliminated by requiring a minimum region size.

Fig. 8 shows the results of one (of many) experiments using the back propagation ANN with both radiometric and textural features to predict and identify slump slides on the levees. Most of the known slides were detected, and some areas of the levee that appear to be suspect but not previously known to have fully developed slides were also flagged as shown. One such area is on the opposite side of the levee from a known slide, leading to speculation (not yet verified) that through-seepage may have occurred there.
Fig. 9 shows a map of the decomposition entropy feature in a levee segment with two slides: one of which was active at the time of the radar flight, the other had been repaired. Not only does the active slide show higher entropy values than the nonslide portions of the levee, but the repaired slide more closely resembles the non-slide areas in having lower entropy values. This feature clearly has potential for our application and will be investigated further along with its related polarimetric decomposition parameters.

As has been stated, these results are still preliminary and cannot yet be rigorously assessed or validated until more samples are tested. Fortunately, just before this paper went to press a gold mine of additional sample data presented itself: the great flood of the spring of 2011 along the Mississippi River.

\section{FUTURE WORK}

Reports from the USACE on the great flood of spring 2011, a 100 year flood event, are being analyzed in conjunction with extra flights of the UAVSAR and extra TerraSAR-X satellite acquisitions corresponding to times near, during, and after the peak flood levels. Though the mainline levees held during this flood (no catastrophic failures), there were many issues with slump slides, through-seepage, and sand boils that had to be quickly repaired by the Corps. The radar signatures corresponding to their locations will analyzed with the algorithms reported on here for further training and validation.

\section{ACKNOWLEDGMENT}

The authors acknowledge the contributions of two partnering organizations in this research: The US Army Corps of Engineers, and Soil and Topography Information, LLC .

\section{REFERENCES}

[1] J. G. Williams, Sand boils: A modern analogue of ancient aand volcanos, Arkansas Academy of Science Proceedings, Vol. XXVI11, 1974, pp 80-81.

[2] J. Dunbar, "USACE's lower Mississippi valley engineering geology and geomorphology mapping program for levees," presentation at Vicksburg, MS, April 16, 2009.

[3] A. K. M. A. Hossain, G. Easson, K. Hasan, "Detection of Levee Slides Using Commercially Available Remotely Sensed Data", Environmental and Engineering Geoscience, v.12; no. 3; pp. 235 - 246, 2006.

[4] Jiancheng Shi, J. Wang, A. Hsu, P. O’Neill, E.T. Engman, "Estimation of bare soil moisture and surface roughness parameter using L-band SAR image data," IEEE Trans. Geoscience Remote Sensing, Vol. 35, No. 5, 1254-1266, 1997

[5] Kevin Wheeler, Scott Hensley, Yunling Lou, Tim Miller, Jim Hoffman, "An L-band SAR for repeat pass deformation measurements on a UAV platform", 2004 IEEE Radar Conference, Philadelphia, PA, April 2004.

[6] Aanstoos, J.V., Hasan, K., Mahrooghy, M., Dabbiru, L., Nobrega, R. A. A., \& Prasad, S. Screening of Earthen Levees Using TerraSAR-X Radar Imagery. 4th TerraSAR-X Science Team Meeting. Oberpfaffenhofen, Germany: DLR (German Aerospace Center), February 2011.

[7] R. O. Duda, P. E. Hart and D. G. Stork, Pattern Recognition, 2nd ed. ed. Hoboken, NJ: Wiley-Interscience, 2000.

[8] Dabbiru, L., \& Aanstoos, J.V. (2010). Classification of Levees using Polarimetric Synthetic Aperture Radar (SAR) Imagery. Proc. 2010 39th IEEE Applied Imagery Pattern Recognition Workshop. Washington, DC: IEEE.

[9] Yisok Oh, "Quantitative retrieval of soil moisture content and surface roughness from multipolarized radar observations of bare soil surfaces," 
Geoscience and Remote Sensing, IEEE Transactions on , vol.42, no.3, pp. 596- 601, March 2004.

[10] Y. Oh, K. Sarabandi, and F. T. Ulaby, "An empirical model and an inversion technique for radar scattering from bare soil surfaces", IEEE Trans. Geosci. Remote Sensing, vol. 30, pp.370 -382 1992.

[11] Mahrooghy, M., Aanstoos, J.V., Hasan, K., \& Younan, N. H. (2011). Levee Soil Moisture Assessment Based On Backpropagation Neural Network Using Synthetic Aperture Radar. Proceedings of the 34th International Symposium for Remote Sensing of Environment. Sydney, Australia.

[12] R.M. Haralick, K. Shanmugam and I. Dinstein. "Textural features for image classification," IEEE Trans. Syst. Man Cybern. 3 (1973), pp. 610-621.

[13] C. S. Burrus, R. A. Gopinath, H. Guo, Introduction to Wavelets and Wavelet Transforms: A Primer, Prentice Hall, August 24, 1997.

[14] Huang, K., Aviyente, S., "Wavelet Feature Selection for Image Classification," Image Processing, IEEE Transactions on, vol.17, no.9, pp. 1709-1720, Sept. 2008.

[15] Aanstoos, J.V., Hasan, K., O'Hara C., Prasad, S., Dabbiru, L., Mahrooghy, M., Nobrega, R. A. A., Lee, M. L., \& Shrestha, B. Use of Remote Sensing to Screen Earthen Levees. Proc. 2010 39th IEEE Applied Imagery Pattern Recognition Workshop. Washington, DC: IEEE.

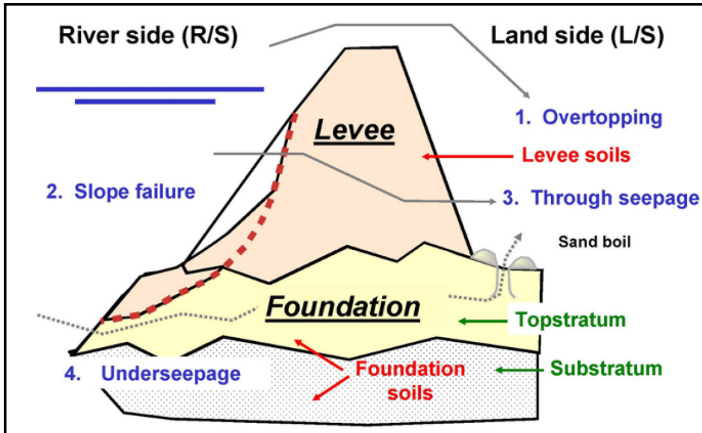

Figure 1. Illustration of levee failure mechanisms, including slough slides and sand boils

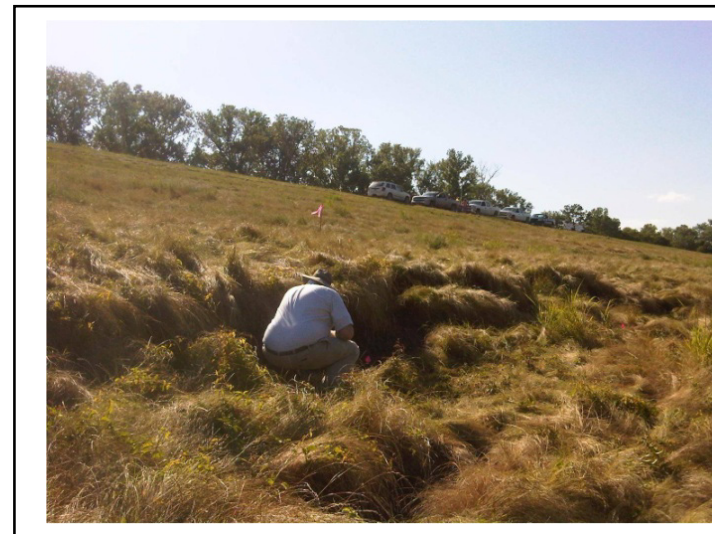

Figure 2. Typical levee slough (or slump) slide. This one appeared during the major flood event in the Spring of 2011

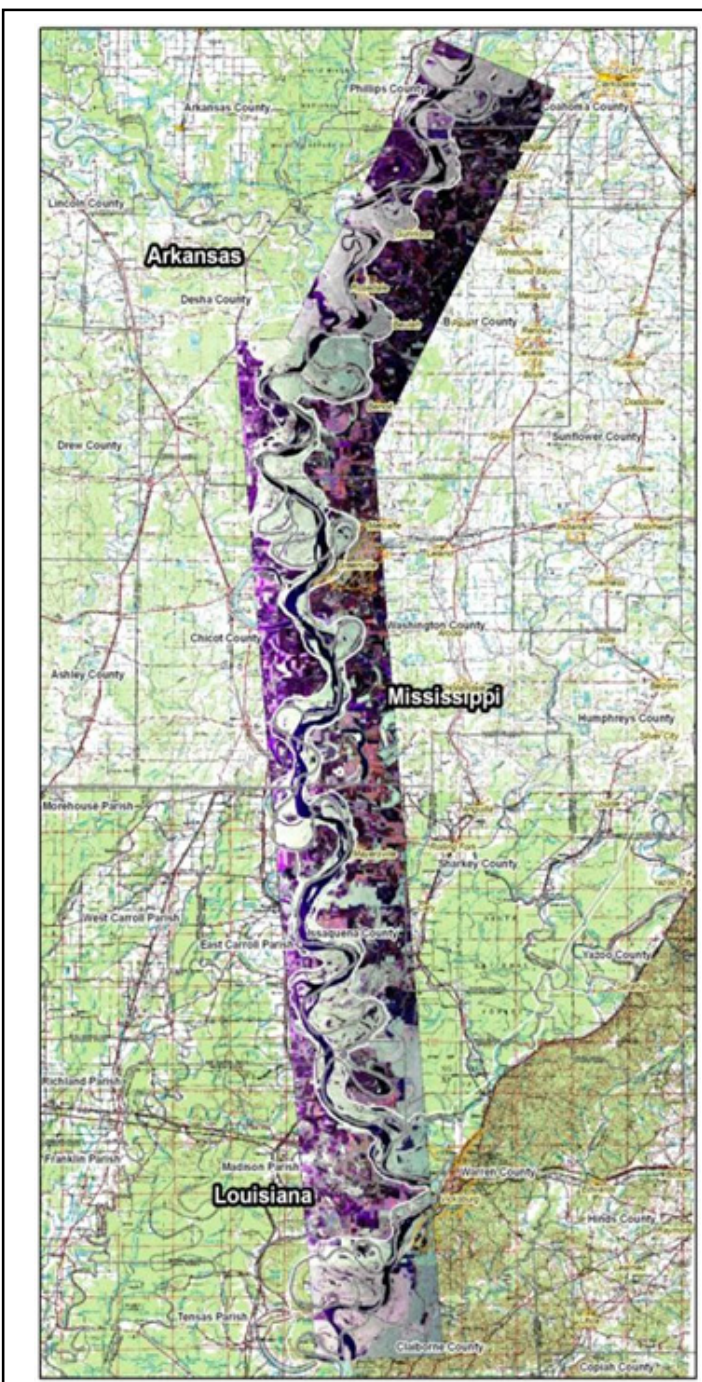

Figure 3. Study area with radar color composite image overlaid on base map. Radar swath is $20 \mathrm{~km}$ wide and total length is $238 \mathrm{~km}$ of the lower Mississippi River valley bordering Arkansas, Mississippi, and Lousisiana.

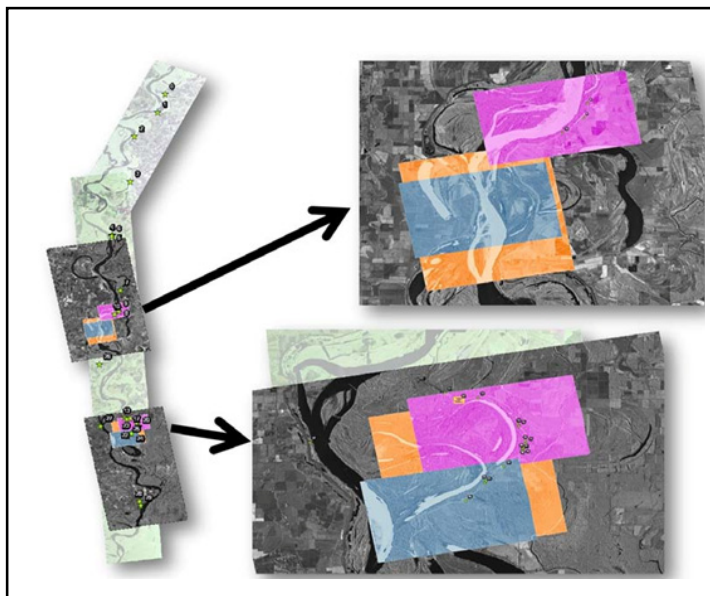

Figure 4. Location of TerraSAR-X satellite SAR scenes in relation to the larger UAVSAR image swath. 


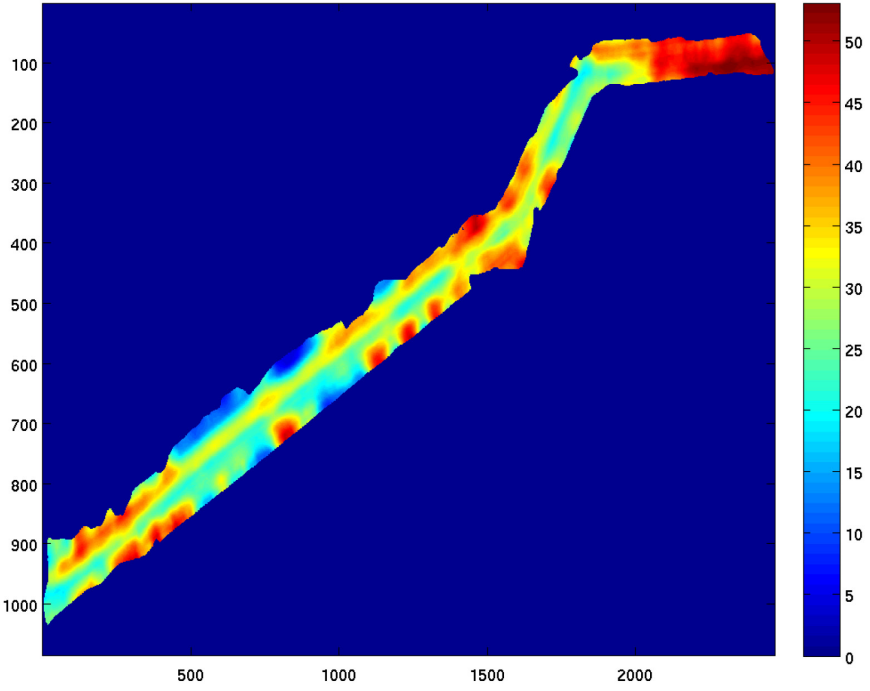

Figure 5. Example of in-situ soil properties data: soil moisture along levees
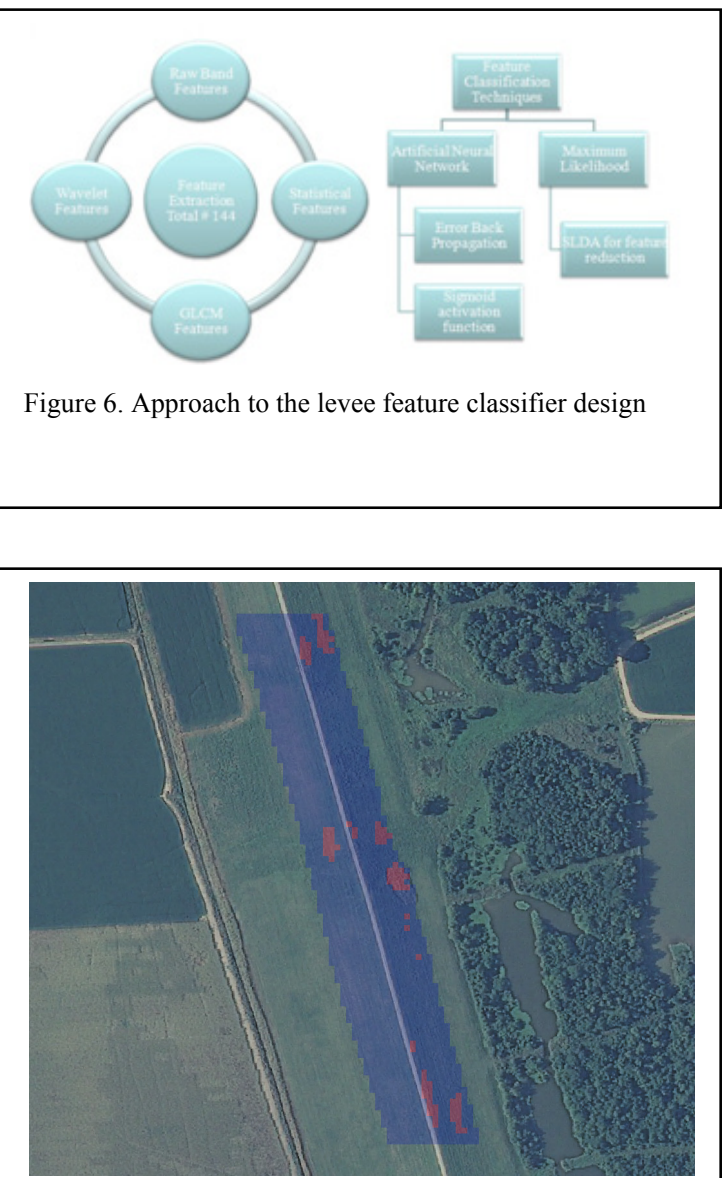

Figure 7. Levee slide classification using best 2 GLCM features selected by S-LDA algorithm

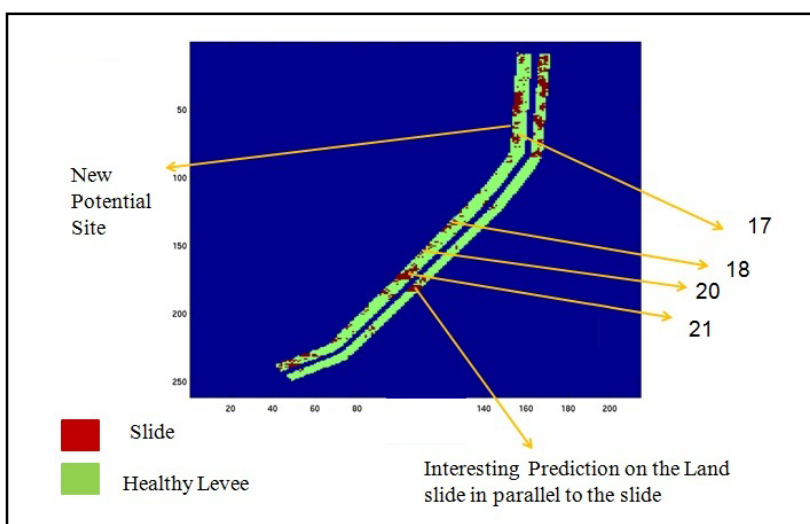

Figure 8. Results of ANN classifier prediction of slump slides on a section of levee, using X-band

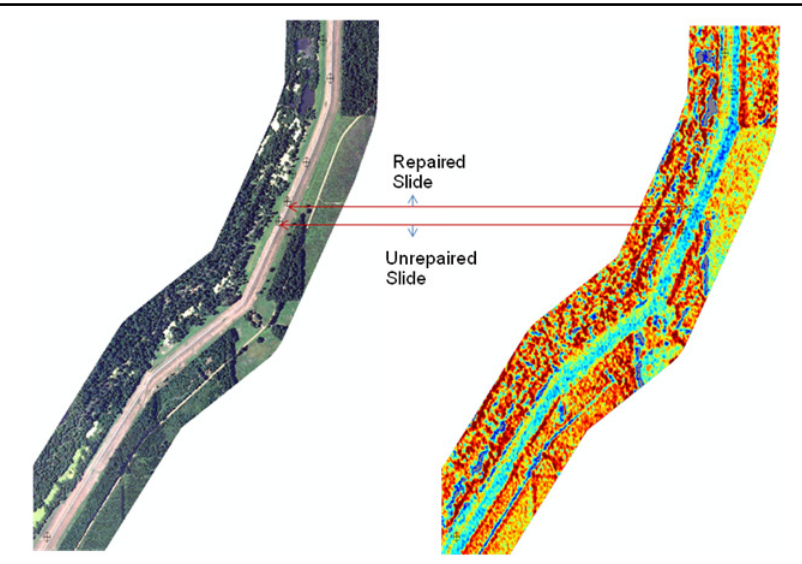

Figure 9. Color-coded mgnitude of Shannon Entropy computed from the L-band UAVSAR data, showing high relative value corresponding to an active slide, with lower values in a nearby slide that had been repaired before the radar acquisition 